\title{
Prefrontal Cortical Projections to the Midbrain in Primates: Evidence for a Sparse Connection
}

\author{
William Gordon Frankle', Mark Laruelle ${ }^{1,2}$ and Suzanne N Haber*,3 \\ 'Department of Psychiatry, Columbia University College of Physicians and Surgeons, New York, NY, USA; '2Department of Radiology, Columbia \\ University College of Physicians and Surgeons, New York, NY, USA; ${ }^{3}$ Department of Pharmacology and Physiology, University of Rochester School \\ of Medicine, Rochester, NY, USA
}

\begin{abstract}
Frontal cortical efferent fibers are thought to have important regulatory influence on cortico-basal ganglia (BG) circuits. The corticomidbrain (substantia nigra/ventral tegmental area, SN/NTA) pathway has received particular attention in psychiatric diseases, most notably schizophrenia. Work in rodents demonstrates that the prefrontal cortico (PFC)-midbrain pathway plays a central role in regulating the firing pattern of dopamine (DA) neurons. These findings have led to some important hypotheses concerning PFC/BG interaction in schizophrenia. Descending PFC projections to the SN/NTA have been primarily documented in the rodent. The aim of this study was to determine the degree and organization of PFC afferents to these areas in the Macaque monkey. Anterograde tracer injections were made into discrete orbital, cingulate, and dorsolateral prefrontal areas. Projections were charted to the SN and VTA. Overall, there were very few fibers in the ventral midbrain following injections confined to specific areas of the PFC. To determine the relationship of the descending fibers to the midbrain DA neurons, sections were double stained for the tracer molecules and for tyrosine hydroxylase. In all cases, the prefrontal projections and the TH-positive cells did not appear to be in close juxtaposition. The results show a very limited projection from the PFC to the midbrain DA neurons in primates, terminating both within the SN proper as well as in the VTA. They arise from a broad region of the PFC, including the DLPF, cingulate, and orbital cortices. However, despite the relative lack of cortical input to the midbrain cells, these neurons are rich in glutamate receptors in primates. Thus, while, based on these anatomical studies, direct cortical control of DA neurons remains debatable in primates; the cortex may directly impact other sources of glutamatergic control.

Neuropsychopharmacology (2006) 3 I, 1627-1636. doi:I 0. I 038/sj.npp. I 300990; published online 4 January 2006
\end{abstract}

Keywords: frontal cortex; substantia nigra; ventral tegmental area; dopamine

\section{INTRODUCTION}

Understanding how the midbrain dopamine (DA) neurons are regulated is critical for gaining insight into the reward system and into the abnormalities seen in diseases such as drug addiction and schizophrenia. Based on rodent studies, cortical control of the midbrain is one important mechanism by which glutamatergic input either directly or indirectly can drive or modulate DA cells (Maurice et al, 1999; Sesack and Carr, 2002). It has been demonstrated with microdialysis that release of DA in the medial striatum can be induced by the infusing the GABA-A receptor antagonist, bicuculline, into the prefrontal cortex (PFC). This effect can be blocked by

*Correspondence: Dr SN Haber, Department of Pharmacology and Physiology, University of Rochester School of Medicine, 60I Elmwood Ave., Rochester, NY |4642, USA, Tel: + I 716275 4538, Fax: + I 716 273 2652, E-mail: Suzanne_Haber@urmc.rochester.edu

Received 2 March 2005; revised 19 October 2005; accepted 24 October 2005

Online publication: 28 October 2005 at http://www.acnp.org/citations/ Npp I02805050 I44/default.pdf the application of excitatory amino-acid receptor antagonists in the ventral tegmental area (VTA) (Karreman and Moghaddam, 1996). More specifically, application of the selective AMPA/kainate antagonist, LY293558, to the VTA reduces DA release in the PFC, but increases DA levels in the nucleus accumbens (NAc) (Takahata and Moghaddam, 2000). These results support dual AMPA-receptor mediated regulation of DA in the VTA; inhibitory regulation of DA neurons projecting to the NAc and excitatory regulation of DA neurons projecting to the PFC. Anatomical studies show that afferents from the PFC innervate the VTA GABAergic cells that project to the NAc (Christie et al, 1985; Sesack et al, 1989a, b; Hurley et al, 1991; Sesack and Pickel, 1992a,b) and the VTA DA cells that project back to the PFC (Carr and Sesack, 2000a,b). In this way, blockade of excitatory stimulation from the PFC to the VTA can produce two opposite results: (1) an increase in $\mathrm{DA}$ in the NAc via a reduction in inhibitory GABA drive to the DA neuron that project to the NAc; and (2) a decrease in $\mathrm{DA}$ in the PFC via a reduction in excitatory drive to DA neuron that project back to the PFC (Sesack and Carr, 2002). 
This cortico-DA interaction supports the 'revised' DA hypothesis of schizophrenia (Weinberger, 1987; Davis et al, 1995). The 'classical' DA hypothesis proposed that there is a hyperactivity of DA transmission, which is responsible for the positive symptoms (Carlsson and Lindqvist, 1963; Seeman and Lee, 1975; Creese et al, 1976). Imaging studies in patients with schizophrenia show increased subcortical DA release in response to an amphetamine challenge and increased baseline subcortical DA levels (Laruelle et al, 1996; Breier et al, 1997; Abi-Dargham et al, 1998, 2000), lending additional support to the hypothesis of increased subcortical DA in schizophrenia. However, recent studies implicate a deficit in DA transmission at $D_{1}$ receptors in the dorsolateral prefrontal cortex (DLPFC) in the cognitive impairments present in patients with schizophrenia (Goldman-Rakic and Selemon, 1997; Goldman-Rakic, 1999; Goldman-Rakic et al, 2000). Impairment of higher cognitive functions, such as working memory, is one of the most enduring symptoms of schizophrenia and a strong predictor of poor clinical outcome (Green, 1996). Furthermore, the negative and cognitive symptoms of the illness are generally resistant to treatment by antipsychotic drugs (Keefe and Gerfen, 1999). Functional brain imaging studies suggesting that these symptoms are associated with a dysfunction of the DLPFC (Manoach et al, 1999, 2000; Callicott et al, 2000) are supported by non-human primates experiments that demonstrate a deficit in DA transmission in the DLPFC and a lack of D1 receptor stimulation induces cognitive impairments similar to those observed in patients (Goldman-Rakic and Selemon, 1997; Goldman-Rakic, 1999; Goldman-Rakic et al, 2000). Thus, the current view of DA and schizophrenia proposes that schizophrenia might be associated with a dopaminergic imbalance, involving an excess of subcortical DA function and a deficit in cortical DA function: DA projections from the midbrain (VTA and substantia nigra (SN)) to subcortical structures might be hyperactive, while mesocortical DA projections to the PFC might be hypoactive.

A general model, based on glutamate modulation of the midbrain DA neurons, for schizophrenia has been proposed by Carlsson et al (1999). This model provides the anatomical framework for the putative neurochemical abnormalities involved in the pathophysiology of schizophrenia including: a deficiency of glutamate transmission, a deficiency of cortical DA transmission and an excess of subcortical DA transmission. However, although the data describe above support cortical glutamatergic control of DA midbrain cells, a direct pathway has been difficult to demonstrate definitively in primates. Previous studies using either fiber degeneration techniques or tritiated amino-acid injections were unable to clearly demonstrate that fibers actually terminated in the ventral midbrain (Leichnetz and Astruc, 1976; Kunzle, 1978). Therefore, using modern anterograde tracers that demonstrate axon terminals, we studied projections from the PFC to the ventral midbrain in the Macaque monkey to determine the extent to which the PFC projects directly to the midbrain. The density of labeled fibers in these midbrain regions was compared to the density of cortical fibers from the same injection sites terminating in the striatum.

\section{MATERIALS AND METHODS}

To examine the organization of prefrontal cortical-midbrain connections, we injected anterograde tracers into the orbital, cingulate, and dorsolateral prefrontal areas. In each case, the distribution and pattern of terminal fibers were charted in both the striatum and midbrain. To determine the relationship of the descending fibers to the midbrain DA neurons, sections were double stained for the tracer molecules and for tyrosine hydroxylase.

\section{Surgery and Tissue Preparation}

In total, 27 adult macaque monkeys (Macaca Nemistrina) were used for these experiments. The University Committee on Animal Resources approved all procedures. Before surgery, monkeys were tranquilized by intramuscular injection of ketamine $(10 \mathrm{mg} / \mathrm{kg})$. Anesthesia was maintained by intravenous injection of pentobarbital (initial dose $20 \mathrm{mg} / \mathrm{kg}$ i.v. and maintained as needed). Temperature, heart rate, and respiration were monitored throughout the surgery. Monkeys were placed in a Kopf stereotaxic, a midline scalp incision was made, and the muscle and fascia were displaced laterally to expose the skull. A craniotomy $\left(\sim 2-3 \mathrm{~cm}^{2}\right)$ was made over the region of interest and small dural incisions only at recording or injection sites. Serial electrode penetrations were performed to determine the dorsal-ventral coordinates for the orbital and cingulate cortical injection sites (see Haber et al, 1993). Dorsal injections sites were determined visually. Accurate placement of tracer injections was achieved by careful alignment of the injection cannulae with the electrode. The dorsolateral injections sites were determined by visual inspection of frontal cortical gyri, indicating general frontal cortical areas.

Monkeys received an injection of one of the following anterograde tracers, Lucifer Yellow, Fluororuby, or Fluorescein conjugated to dextran amine (LY, FR, or FS; $40-50 \mathrm{nl}$, $10 \%$ in $0.1 \mathrm{M}$ phosphate buffer (PB), $\mathrm{pH} 7.4$; Molecular Probes, Eugene, OR, USA), or Phaseolus vulgaris-leucoagglutinin (PHA-L; 50nl, 2.5\%; Vector laboratories). Tracers were pressure-injected over $10 \mathrm{~min}$ using a $0.5 \mu \mathrm{l}$ Hamilton syringe. Following each injection, the syringe remained in situ for 20-30 min. At 12 to 14 days post-op, monkeys were again deeply anesthetized and perfused with saline followed by a $4 \%$ paraformaldehyde/ $1.5 \%$ sucrose solution in $0.1 \mathrm{M} \mathrm{PB}, \mathrm{pH}$ 7.4. Brains were postfixed overnight and cryoprotected in increasing gradients of sucrose $(10,20$, and $30 \%)$. Serial sections of $50 \mu \mathrm{m}$ were cut on a freezing microtome into $0.1 \mathrm{M}$ PB or cryoprotectant solution.

\section{Immunocytochemistry}

Immunocytochemistry was performed on free-floating sections to visualize LY, FR, FS, and PHA-L tracers. Prior to incubation in primary antisera, tissue was treated with $10 \%$ methanol and $3 \%$ hydrogen peroxide in $0.1 \mathrm{M} \mathrm{PB}$ to inhibit endogenous peroxidase activity, and rinsed $1-2 \mathrm{~h}$ in PB with $0.3 \%$ Triton X-100 (TX, USA from Sigma). Sections were preincubated in $10 \%$ normal goat serum (NGS) and $0.3 \%$ TX in PB for $30 \mathrm{~min}$. Tissue was placed in the primary antisera-anti-LY (1:2000 dilution, Molecular Probes, Eu- 
gene, OR, USA), anti-FS ( $1: 1000$; Molecular Probes, Eugene, OR, USA), anti-FR $(1: 1000$; Molecular Probes, Eugene, OR, USA), or anti-PHA-L (1:500; EY Laboratories, Inc.) in $10 \%$ NGS and $0.3 \% \mathrm{TX}$ in $\mathrm{PB}$ for four nights at $4{ }^{\circ} \mathrm{C}$. Following extensive rinsing, the tissue was incubated in the avidin-biotin complex solution (Vectastain $\mathrm{ABC}$ kit, Vector Labs). Immunoreactivity was visualized using standard $\mathrm{DAB}$ procedures. Staining was intensified by incubating the tissue for 5 to $15 \mathrm{~min}$ in a solution of $0.05 \% \quad 3,3^{\prime}$-diaminobenzidine tetra-hydrochloride, $0.025 \%$ cobalt chloride, $0.02 \%$ nickel ammonium sulfate, and $0.01 \% \mathrm{H}_{2} \mathrm{O}_{2}$ to yield a black reaction product. Sections were mounted onto gel-coated slides, dehydrated, defatted in xylenes, and coverslipped with Permount. Sections that were double labeled were first processed for the tracer and then incubated with antisera to TH (1:20000 Eugene Tech, Inc.) (ADD fluorescence section).

\section{Analysis}

Cortical injections with contamination or weak labeling were eliminated from the analysis. Fiber distributions in the midbrain were charted using a Nikon research microscope with brightfield/darkfield illumination, equipped with an imaging system, and Neurolucida software by MicroBrightField. Individual charts were imported into Adobe Illustrator to create composite images. Cortical experiments employed multiple tracers molecules (FS, FR, LY, and PHA-L) that have intrinsic differences in uptake and transport properties. Injections of different tracer molecules into the same site demonstrated similar projection patterns and relative densities between the striatum and midbrain. Therefore, the comparisons between distribution densities between cortico-striatal and cortico-midbrain projections are critical for illustrating the relative influence, based on fiber density. We determined the boundaries of the SN and VTA using counterstained or adjacent, Nissl-stained coronal sections (Figure 1).

\section{RESULTS}

In total, 29 anterograde tracer injections were placed into discrete regions of PFC. The injection sites encompassed three main regions: orbital, cingulate and DLPFC cortex. There were nine cases of orbital prefrontal injections, areas, $14,11,13$, and 12 (orbital proisocortex); seven cases of cingulate injections that targeted areas 24, 32, and 25; and 13 cases with injection sites in the DLPFC that included areas 9 and 46. The injection sites selected for analysis and illustration were all confined to the orbital, DLPF, or cingulate cortices. We selected six cases, two from each general region, cingulate (areas 24 and 25; cases 36 and 83 respectively) orbital (areas 13 and 12, cases 133 and 115 respectively), dorsolateral (areas 9 and 46, cases 106 and 121 , respectively) to illustrate the projections throughout the midbrain. A representative section was also illustrated for three additional cases in each general region (cingulate area 32 , case 124; OPFC 14, case 47; DLPFC area 46, case 131). When more than one case was available for a particular injection site, the case containing the largest projection was selected for illustration. Given the sparse

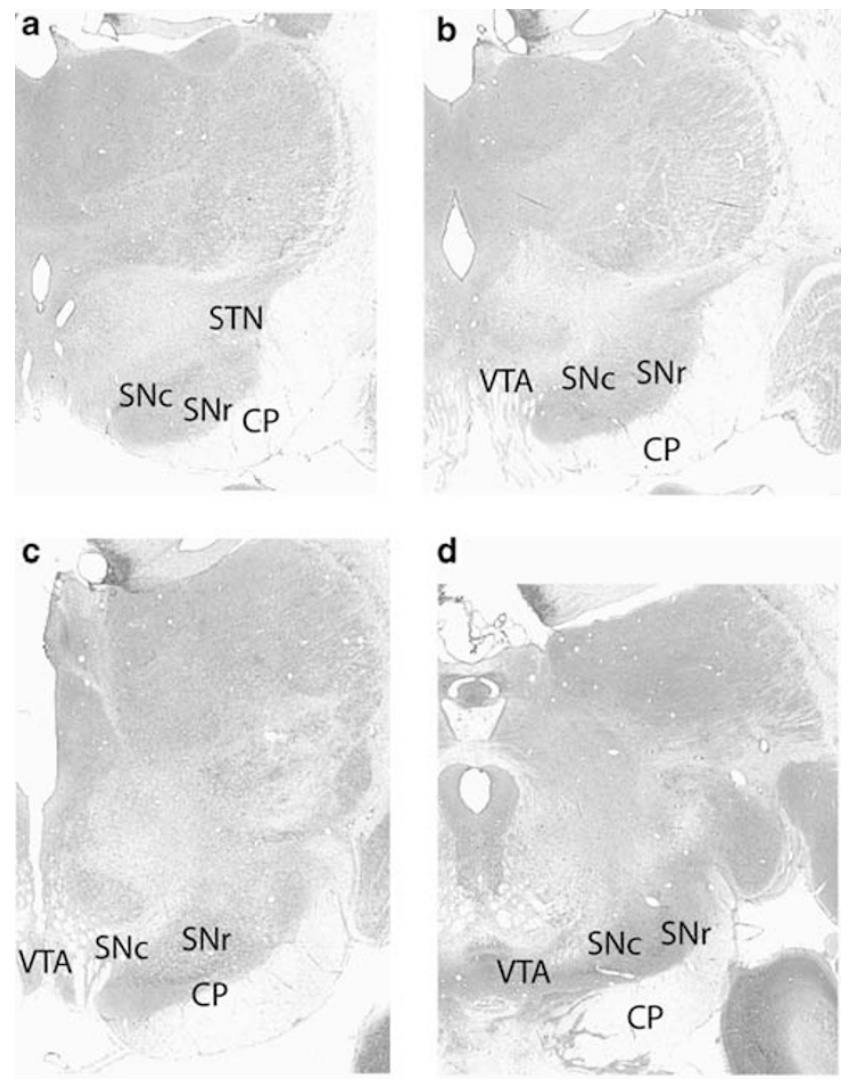

Figure I Photomicrographs of Nissl stained coronal sections through the brainstem of the macaque monkey to illustrate the regions in which descending cortical fibers were charted. CP, cerebral peduncle; SNc, substantia nigra pars compacta; SNr, substantia nigra pars reticulata; STN, subthalamic nucleus; VTA, ventral tegmental area.

nature of the projection fibers in all cases, each fiber in each section was illustrated. Terminal fibers were determined by the presence of fine arborizations and terminal boutons. The terminal fields also contained scattered larger fibers, lacking evidence of termination. These fibers were assumed to be fibers of passage and were not illustrated. However, if there was doubt about whether a fiber was terminating or passing, we chose to illustrate it, given the relatively few fibers present in the projection fields. Therefore, if an error exists, it is on the side of over representation.

In general, as indicated by the illustrations, overall there are very few fibers in the ventral midbrain following small injections confined to specific areas of PFC. In contrast, these injections produced very dense labeling in the striatum, as illustrated for comparison, and in the thalamus (not illustrated). Projections to the midbrain remained ipsilateral to the injection site and demonstrated some topography. The ventral cingulate and orbital cortical areas projected primarily to the VTA and the medial SN. In contrast, the dorsolateral prefrontal and dorsal cingulate cortices projected primarily to the SN.

\section{Cingulate Projections (Figure 2)}

The injection sites in the cingulate $\mathrm{PFC}$ are represented by cases 36,124 , and 83 . Case 36 was an injection of LY in area 
a

Subgenual Cingulate - PHA-L in area 25

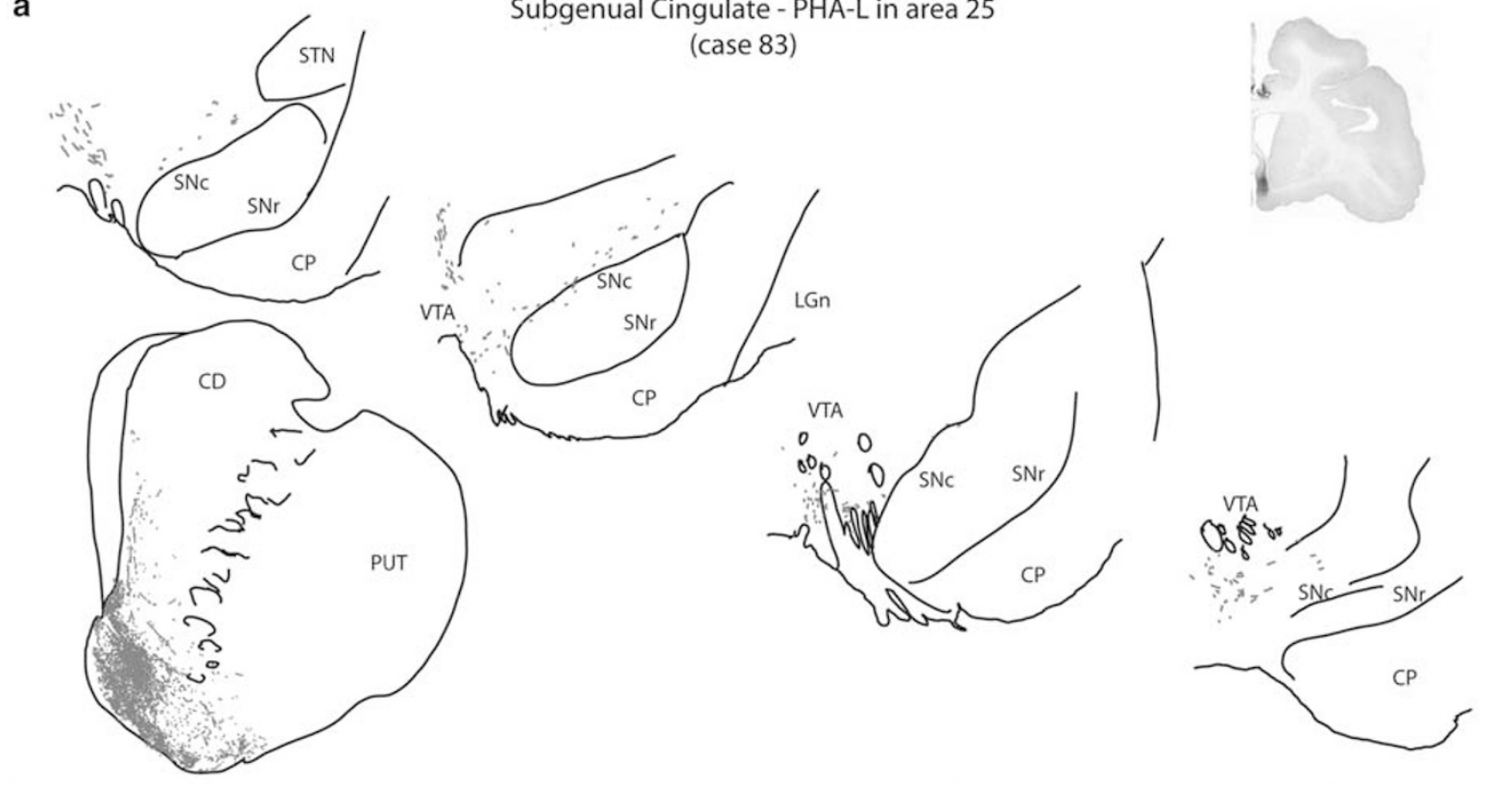

b Cingulate Cortex - FR in area 32

(case 124)

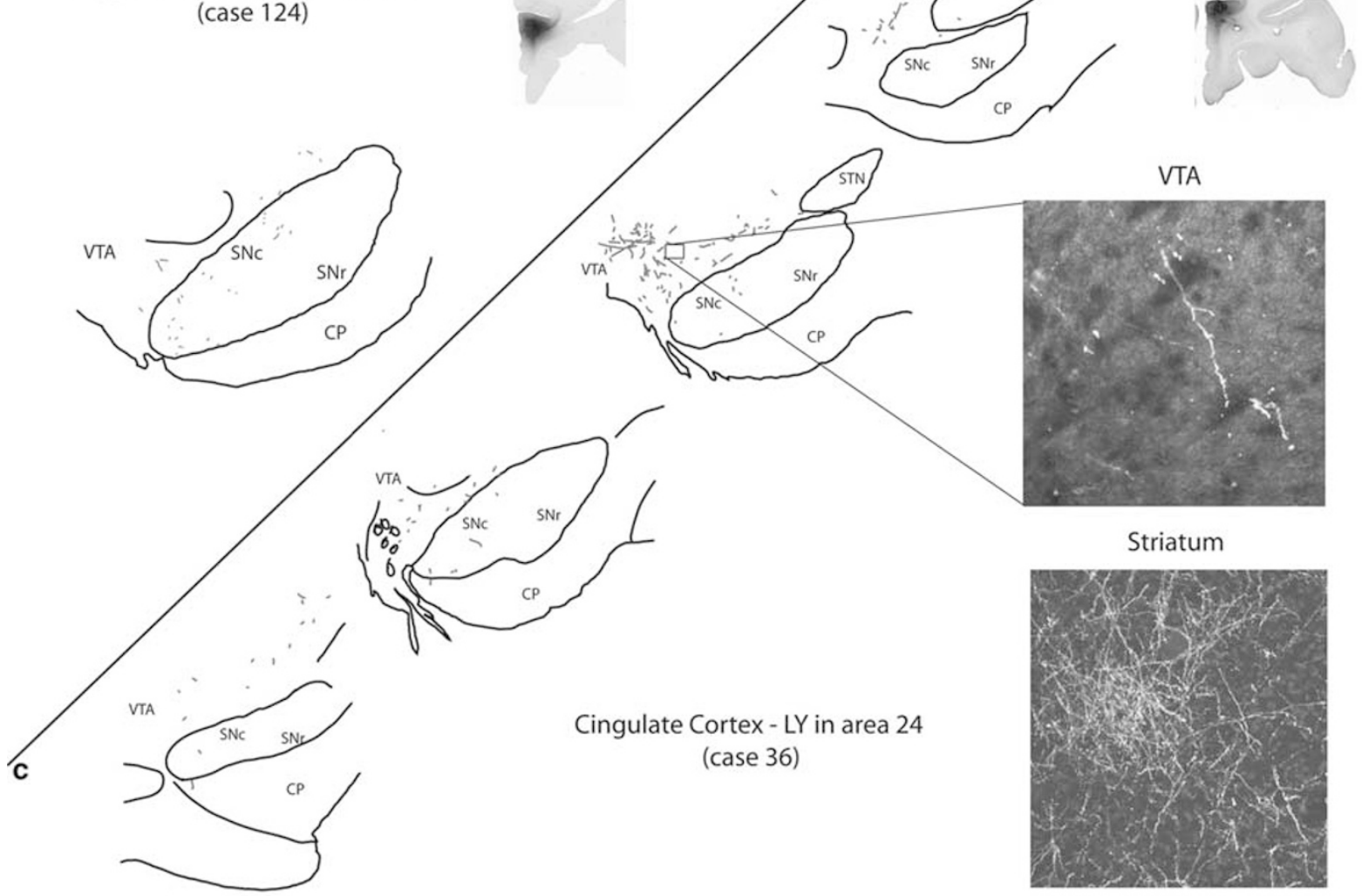

Figure 2 Camera lucida drawings of transverse sections through the midbrain of macaque monkeys illustrating the distribution of anterograde labeled fibers from cingulate cortical regions. Injection sites, shown in the inset photomicrographs, were localized to areas 25, 32, and 24 (a, b, and c, respectively). For comparison, anterograde labeled fibers in a section through the basal forebrain at the level of the striatum were illustrated for the injection site in area 25 (a). In (c), photomicrographs of cortical terminal field in the striatum and in the midbrain following are shown. CD, caudate; CP, cerebral peduncle; PUT, putamen; SNc, substantia nigra pars compacta; SNr, substantia nigra pars reticulata; STN, subthalamic nucleus; VTA, ventral tegmental area.

24 , case 124 an injection of FR in area 32 , and case 83 an injection of PHAL in area 25 . The number of projection fibers from these injection sites was somewhat greater than that seen with injections into the orbital PFC (see below and Figure 3). However, relative to the dense projections seen in the striatum from these same injections, the total projection 
remains very sparse. Fibers projected largely to the VTA and retrorubral area. Following an injection into area 25 individual fibers were noted in the VTA throughout the midbrain. There were no fibers in the region of the SN. In contrast, following injections into areas 24 and 32, there were fibers both in the VTA and in the medial and dorsal $\mathrm{SN}$. All injections resulted in very dense, topographically organized terminal fields in both the striatum and thalamus.

\section{Orbital Prefrontal Projections (Figure 3)}

Case 115 was an injection of LY into area Opro (orbital proisocortex), case 133 represents an injection of FR into area 11 , and case 47 was an injection of LY into area medial 14. There were isolated labeled fibers at all levels of the midbrain in each case. Furthermore, descending fibers continued caudally and were noted in the other brainstem regions, including the central grey. In contrast to the sparse labeling in the midbrain, the striatum had very dense topographically organized terminals (Figure 3 ). Following the injection into Opro, labeled fibers were found primarily in the SN, with few labeled fibers in the VTA. At rostral levels, labeled fibers were located in the medial and dorsal SN; however, unlike the other cases, at caudal levels, they were also observed more ventrally. Following an injection of FR into area 11, the labeled fibers were located primarily within the SN proper, in the medial and dorsal region. However, compared to case 115, there were more labeled fibers in the VTA, particularly at rostral levels. Case 47 , an injection into area 14 was the most medial case of the orbital prefrontal cortices. This injection resulted in the greatest number of labeled fibers in the VTA, extending dorsal to the $\mathrm{SN}$ in the region of the retrorubral cell group. In this case, there were relatively few labeled terminals in the SN, pars compacta. Overall, projections from orbital prefrontal areas yielded minimal projection fibers in the midbrain and were distributed somewhat topographically. The injection sites in the ventral-medial orbital cortex producing labeled fibers primarily in the VTA. Injection sites placed more laterally gave rise to fibers distributed within the SN proper.

\section{Dorsolateral Prefrontal Projections (Figure 4)}

Projections from the dorsolateral PFC were assessed by injections into areas 9 and 46. Representative cases from these injection sites are, case 106 for area 9, case 121 primarily area 46, with some inclusion of area 9 and case 131 for area 46. Injections into areas 9 and 46 resulted in labeled fibers seen primarily in the medial and more dorsal aspect of the SN, with fewer fibers in the VTA. This was similar to our observations from the other cases with injections in this region (data not shown). Case 121, which was a relatively large injection site compared to the other injections, resulted projection fibers localized to the medial and dorsal aspect of the SN. Labeled fibers were concentrated in the central part, with fewer labeled fibers in the most rostral and caudal regions. Despite the relatively large injection site, the density of labeled fibers in the midbrain remained low and, as with the other cases, each fiber is illustrated.

\section{Relationship with TH-Positive Cells}

Double staining for $\mathrm{TH}$ and tracer was performed to examine the relationship between the cortical projection fibers and TH-positive cell bodies and processes. Examination of all cases yielded similar results. Positively stained projections from prefrontal areas could be visualized interspersed with TH-positive cells and processes in both the substantia nigra pars compacta and pars reticulata. However, in all cases, the prefrontal projections and the THpositive cells did not appear to be in close juxtaposition. There were no sections in which a prefrontal projection fiber and a TH-positive fiber were visualized in the same $z$-axis plane (Figure 5 ).

\section{DISCUSSION}

The results show a very limited projection from the PFC to the midbrain DA neurons in primates, terminating both within the SN proper as well as in the VTA. They arise from a broad region of the PFC, including the DLPF, cingulate, and orbital cortices. It is difficult to compare the density of projections from different cortical areas to the midbrain, given the scarcity of this projection. This is particularly apparent when compared to the density of the other descending projections of the prefrontal region, particularly to the striatum and thalamus.

Although no other studies have specifically examined projections from the PFC to the midbrain DA neurons in primates, several authors have reported on PFC projections to this area in the context of studying projections to other brain regions. A set of two publications by the same group which described the prefrontal projections to the hypothalamus and the periaqueductal gray in the macaque monkey show results similar to the current study, with a limited number of labeled axons in the mesencephalon resulting from injections in the PFC (An et al, 1998; Öngür et al, 1998). Consistent with the findings from anterograde studies, retrograde tracer injections into the SN pars compacta and the VTA result in some labeling in medial cingulate cortex (see Figure 5 in Öngür et al, 1998). Freedman et al (2000) studied the subcortical projections from area 25 and, although they did not comment specifically on termination of fibers in the SN and VTA, they noted fibers passing dorsal to the SN pars compacta giving rise to collaterals with few varicosities that pass through the VTA (see Figure 4c). In a similar study, efferent projections from areas $25,24,32$, 10 , and 14 showed some projections to the VTA from areas 25,24 , and 32 , with the strongest projection from area 25 (Chiba et al, 2001).

The findings in rodents differ somewhat from that is seen in the primate. In rodents, a region of the cortex, analogous to the primate PFC, that represents a multimodal association area, separate from the motor and premotor areas, is defined based upon thalamocortical and corticocortical connectivity (Reep et al, 1984; Van Eden et al, 1992; Groenewegen et al, 1997). The rodent PFC is subdivided into a ventromedial area, comprising the infralimbic, prelimbic and anterior cingulate areas, and a dorsocentral region comprised largely by the medial agranular cortex (McGeorge and Faull, 1989; Berendse 

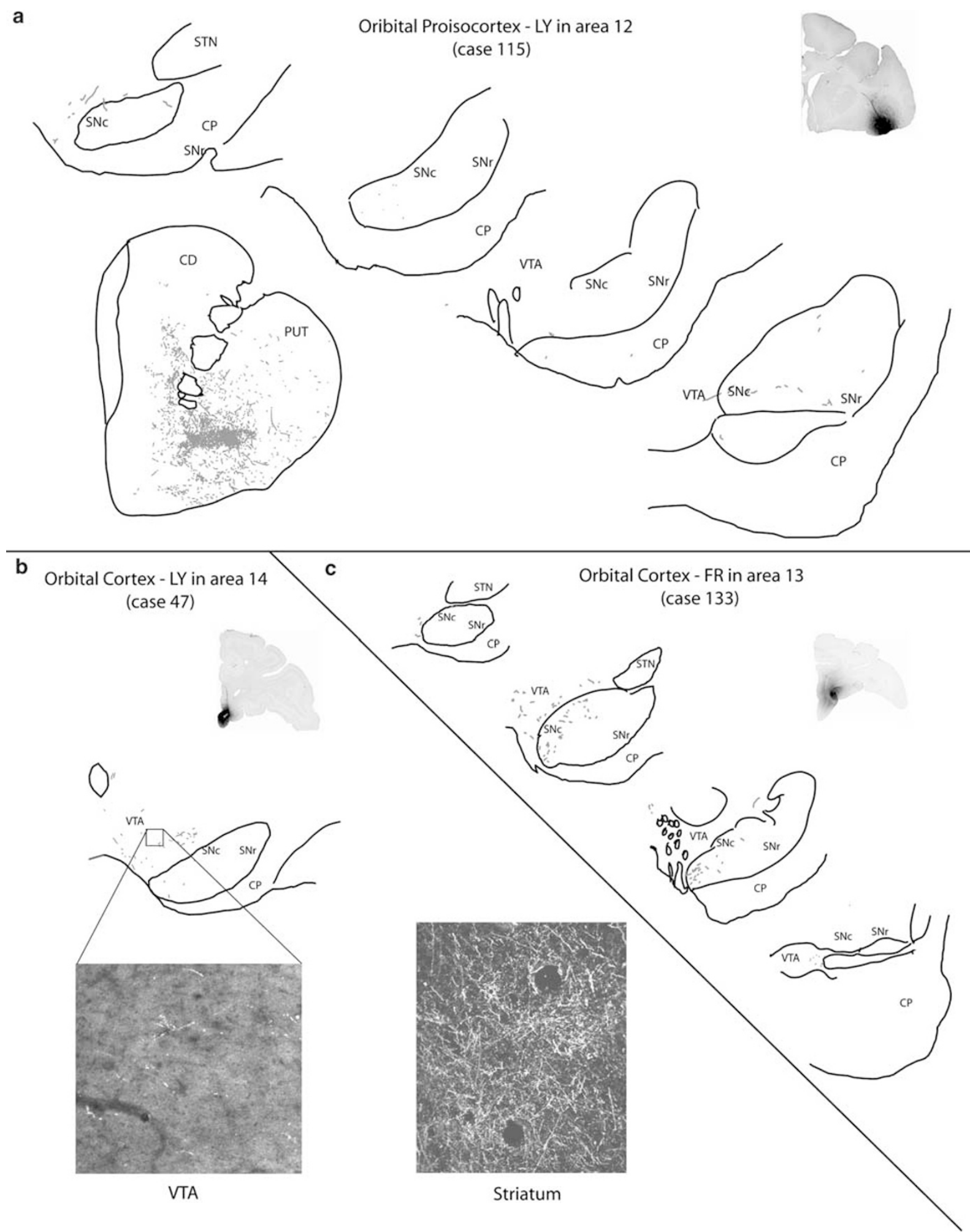

Figure 3 Camera lucida drawings of transverse sections through the midbrain of macaque monkeys illustrating the distribution of anterograde labeled fibers from the orbital frontal cortical regions. Injection sites, shown in the inset photomicrographs, were localized to areas 12, 14, and 13 (a, b, and c, respectively). For comparison, anterograde labeled fibers in a section through the basal forebrain at the level of the striatum were illustrated for the injection site in area 12 (a). In (b), photomicrographs of cortical terminal field in the striatum and in the midbrain following are shown. CD, caudate; CP, cerebral peduncle; PUT, putamen; SNc, substantia nigra pars compacta; SNr, substantia nigra pars reticulata; STN, subthalamic nucleus; VTA, ventral tegmental area.

et al, 1992). In contrast to the weak projections observed in the primate, there is a relatively dense projection from the prelimbic and infralimbic areas to the midbrain (Sesack et al, 1989a, b; Hurley et al, 1991; Takagishi and Chiba, 1991). The discrepancy in the degree of projections from the PFC to the midbrain between the rat and the primate is 
a

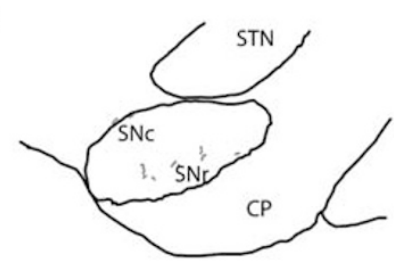

Dorsolateral Prefrontal Cortex - FR in area 46

(case 121)

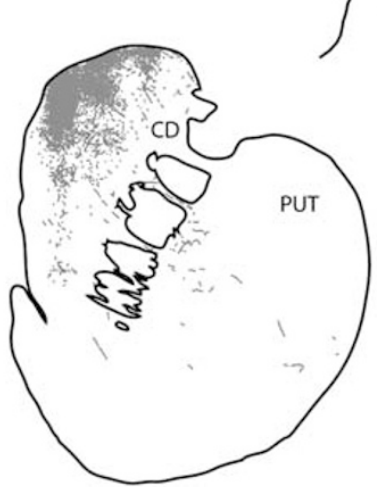

$\mathrm{CP}$
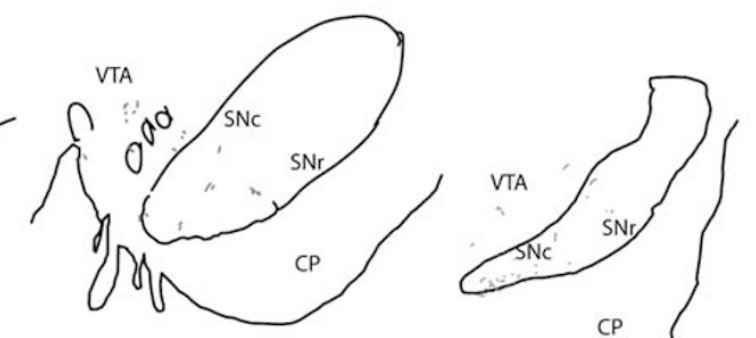

CP
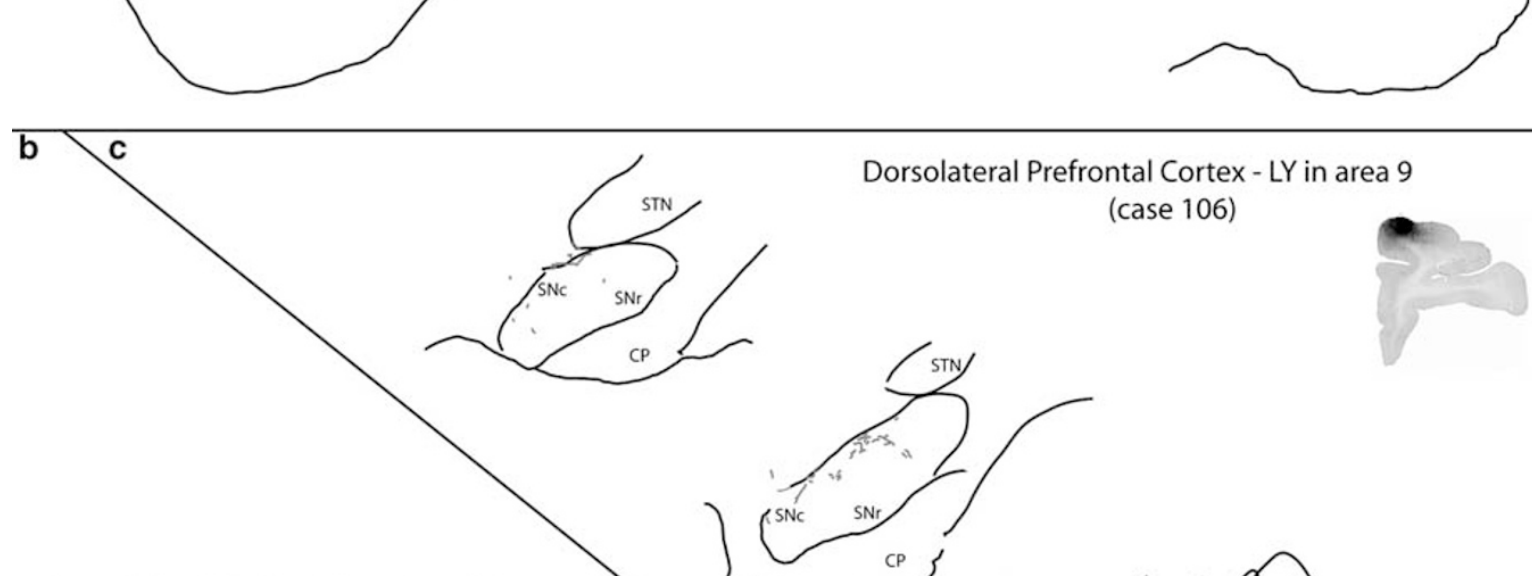

Dorsolateral Prefrontal Cortex - $\mathrm{LY}$ in area 46 (case 131)

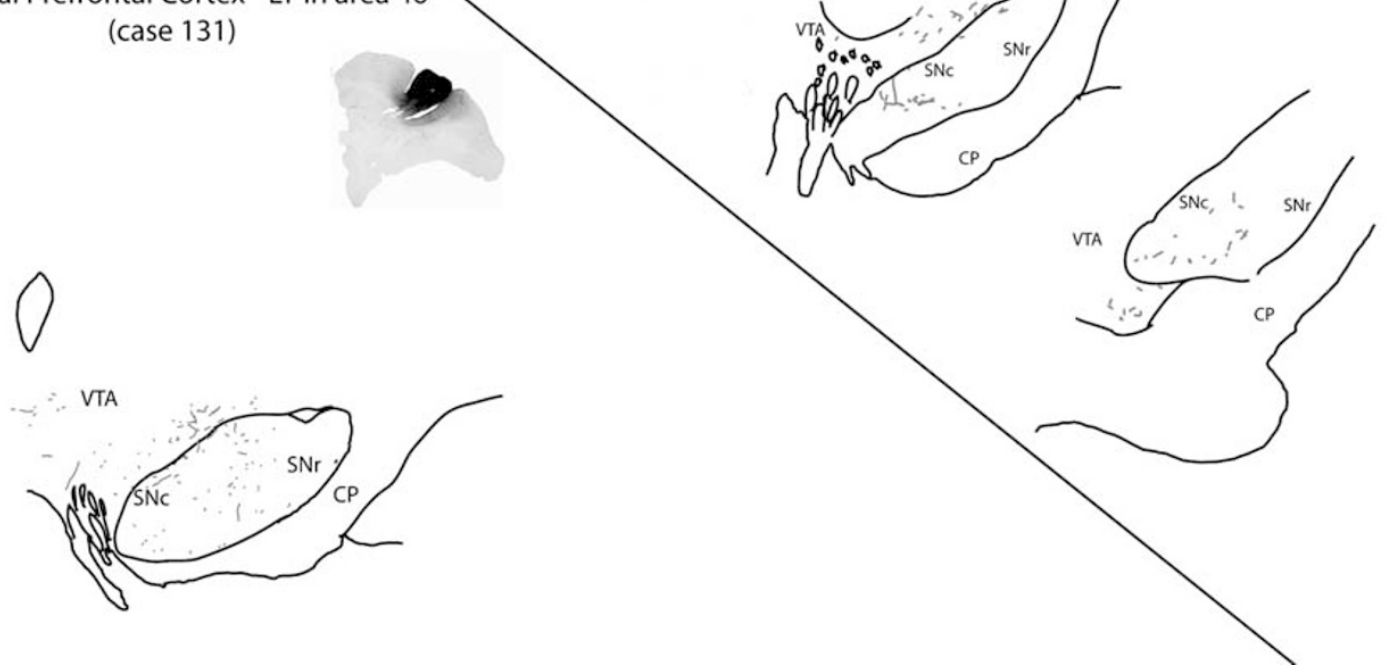

Dorsolateral Prefrontal Cortex $-\mathrm{LY}$ in area 9 (case 106)

Figure 4 Camera lucida drawings of transverse sections through the midbrain of macaque monkeys illustrating the distribution of anterograde labeled fibers from the dorsolateral prefrontal cortex. Injection sites, shown in the inset photomicrographs, were localized to areas 46 and 9 (b and c, respectively) with a large injection in area 46 with some encroachment into area 9 (a). For comparison, anterograde labeled fibers in a section through the basal forebrain at the level of the striatum were illustrated for the injection site in area 46/9 shown in (a). CD, caudate; CP, cerebral peduncle; PUT, putamen; SNc, substantia nigra pars compacta; SNr, substantia nigra pars reticulata; STN, subthalamic nucleus; VTA, ventral tegmental area.

interesting given that in both species projections to other regions, such as the striatum and thalamus are dense. With the large cortical expansion in primates compared to rodents, it is possible that larger injection sites within PFC are needed to appreciate the extent of the projection to the midbrain. 


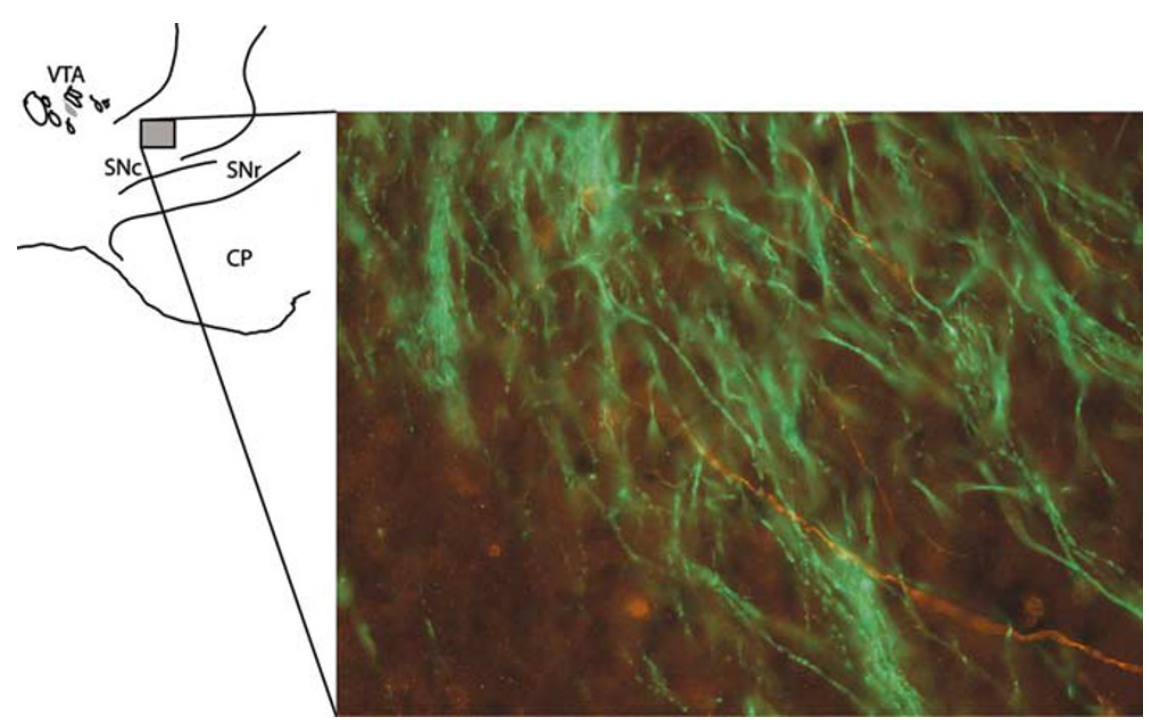

Figure 5 A schematic diagram of the ventral midbrain with a florescent photomicrograph from an individual case in which the anterograde tracer LY was placed in the prefrontal cortex. The section is double-stained for TH (green) and LY (red). CP, cerebral peduncle; SNc, substantia nigra pars compacta; SNr, substantia nigra pars reticulata; VTA, ventral tegmental area.

The importance of direct control of DA function by glutamatergic cells in the PFC has been shown in rodents (Afifi et al, 1974; Bunney and Aghajanian, 1976; Carter, 1980; Kornhuber, 1984; Sesack et al, 1989a, b; Hurley et al, 1991; Sesack and Pickel, 1992a,b). The specificity of synaptic connections between the PFC and the VTA/SN may indicate that a large projection may not be necessary for the PFC to exert significant control over the relevant midbrain cells (Carr and Sesack, 2000a,b). While our findings do not show an extensive anatomical basis for direct PFC control of the VTA or SN DA cells, it is possible that a relatively small projection can indeed have a large affect. However, it is important to note that we were unable to visualize any projection fibers in close apposition with TH-positive cells or processes. Taken together, the results raise the issue of possible alternative pathways for PFC glutamatergic control of DA.

Despite the relative lack of cortical input to the midbrain cells, these neurons are rich in glutamate receptors in primates (Paquet et al, 1997). Thus, while, based on these anatomical studies, direct cortical control of DA neurons remains debatable in primates; the cortex may directly impact other sources of glutamatergic control. The main input to the DA cells (either directly or indirectly) is from the striatum. Other sources of regulation of DA are derived from the globus pallidus (GP), the pedunculopontine n. (PPN), and the subthalamic n. (STN) (Carpenter et al, 1981a,b; Parent, 1986; Smith et al, 1990; Rosales et al, 1994; Charara et al, 1996; Haber, 2003). Of these inputs, both the striatal and pallidal afferent fibers to the SN are GABAergic. In contrast, the STN and the PPN are known to be glutamatergic (for a review see Parent, 1986; Smith et al, 1998; for a review see Haber, 2003). In addition, recent evidence indicates that input to the SN from the bed $\mathrm{n}$. of the stria terminalis (Fudge and Haber, 2001) is also glutamatergic (Georges and Aston-Jones, 2002; Dong and Swanson, 2004).

Among the alternative pathways for excitatory control of the DA neurons, the prefrontal cortical influence via the STN on basal ganglia output structures is of particular recent interest. The frontal cortex provides a main and topographically organized, monosynaptic, glutamatergic input to the STN (Monakow et al, 1978; Carpenter et al, 1981a, b; Nambu et al, 1996; Nambu et al, 1997). The cortico-STN projection, which has been a focus of study for motor and premotor cortico-basal ganglia system, is termed the 'hyperdirect' basal ganglia pathway in that impulses reach the basal ganglia output nuclei faster via this route than through the cortico-striatal pathway (Kolomiets et al, 2003). Given that the STN impacts directly on DA cells (Rosales et al, 1994; Meissner et al, 2003), this route (PFC to the STN to the midbrain cells) may represent an alternative in the primate to the direct PFC-VTA/SN pathway observed in other species. In fact, PFC stimulation results in both excitatory and inhibitory responses in the primate $\mathrm{SN}$ pars reticulata, which are mediated via the STN and striatum, respectively (Kitano et al, 1998). The PFC-STN connection in primates (Frankle et al, 2003), along with the direct STN connection to the $\mathrm{SN}$, is likely to have an impact not only on the SN pars reticulata cells, but also on the DA neurons (Rosales et al, 1994).

\section{ACKNOWLEDGEMENTS}

We thank April Whitbeck and Brian Bouverat for their excellent technical assistance. This work was supported by the Lieber Center for Schizophrenia Research at Columbia University College of Physicians \& Surgeons and MH45573 to $\mathrm{SNH}$.

\section{REFERENCES}

Abi-Dargham A, Gil R, Krystal J, Baldwin RM, Seibyl JP, Bowers M et al (1998). Increased striatal dopamine transmission in schizophrenia: confirmation in a second cohort. Am J Psychiatry 155: 761-767.

Abi-Dargham A, Rodenhiser J, Printz D, Zea-Ponce Y, Gil R, Kegeles LS et al (2000). From the cover: increased baseline 
occupancy of D2 receptors by dopamine in schizophrenia. Proc Natl Acad Sci USA 97: 8104-8109.

Afifi AK, Bahuth NB, Kaelber WW, Mikhael E, Nassar S (1974). The cortico-nigral fibre tract. An experimental Fink-Heimer study in cats. J Anat 118: 469-476.

An X, Bandler R, ngür D, Price JL (1998). Prefrontal cortical projections to longitudinal columns in the midbrain periaqueductal gray in macaque monkeys. J Comp Neurol 401: 455-479.

Berendse HW, Galisde Graaf Y, Groenewegen HJ (1992). Topographical organization and relationship with ventral striatal compartments of prefrontal corticostriatal projections in the rat. J Comp Neurol 316: 314-347.

Breier A, Su TP, Saunders R, Carson RE, Kolachana BS, deBartolomeis A et al (1997). Schizophrenia is associated with elevated amphetamine-induced synaptic dopamine concentrations: evidence from a novel positron emission tomography method. Proc Natl Acad Sci USA 94: 2569-2574.

Bunney BS, Aghajanian GK (1976). The precise localization of nigral afferents in the rat as determined by a retrograde tracing technique. Brain Res 117: 423-435.

Callicott J, Bertolino A, Mattay V, Langheim F, Duyn J, Coppola R et al (2000). Physiological dysfunction of the dorsolateral prefrontal cortex in schizophrenia revisited. Cereb Cortex 10: 1078-1092.

Carlsson A, Lindqvist M (1963). Effect of chlorpromazine or haloperidol on formation of 3-methoxytyramine and normetanephrine in mouse brain. Acta Pharmacol Toxicol 20: 140-144.

Carlsson A, Waters N, Carlsson ML (1999). Neurotransmitter interactions in schizophrenia - therapeutic implications. Biol Psychiatry 46: 1388-1395.

Carpenter MB, Baton III RR, Carleton SC, Keller JT (1981). Interconnections and organization of pallidal and subthalamic nucleus neurons in the monkey. J Comp Neurol 197: 579-603.

Carpenter MB, Carlton SC, Keller JT, Conte P (1981). Connections of the subthalamic nucleus in the monkey. Brain Res 224: 1-29.

Carr DB, Sesack SR (2000a). Projections from the rat prefrontal cortex to the ventral tegmental area: target specificity in the synaptic associations with mesoaccumbens and mesocortical neurons. J Neurosci 20: 3864-3873.

Carr DB, Sesack SR (2000b). Projections from the rat prefrontal cortex to the ventral tegmental area: target specificity in the synaptic associations with mesoaccumbens and mesocortical neurons. J Neurosci 20: 3864-3873.

Carter CJ (1980). Glutamatergic pathways from the medial prefrontal cortex to the anterior striatum, nucleus accumbens and substantia nigra. Br J Pharmacol 70: 50-51.

Charara A, Smith Y, Parent A (1996). Glutamatergic inputs from the pedunculopontine nucleus to midbrain dopaminergic neurons in primates: Phaseolus vulgaris-Leucoagglutinin antereograde labeling combined with postembedding glutamate and GABA immunohistochemistry. J Comp Neurol 364: 254-266.

Chiba T, Kayahara T, Nakano K (2001). Efferent projections of infralimbic and prelimbic areas of the medial prefrontal cortex in the Japanese monkey, Macaca fuscata. Brain Res 888: 83-101.

Christie MJ, Bridge S, James LB, Beart PM (1985). Excitotoxin lesions suggest an aspartatergic projection from rat medial prefrontal cortex to ventral tegmental area. Brain Res 333: 169-172.

Creese I, Burt DR, Snyder SH (1976). Dopamine receptor binding predicts clinical and pharmacological potencies of antischizophrenic drugs. Science 19: 481-483.

Davis KA, Gdowski GT, Voigt HF (1995). A statistically based method to generate response maps objectively. J Neurosci Methods 57: 107-118.

Dong HW, Swanson LW (2004). Organization of axonal projections from the anterolateral area of the bed nuclei of the stria terminalis. J Comp Neurol 468: 277-298.
Frankle W, Bennett S, Laruelle M, Haber S (2003). Prefrontal Cotical Projections to the Subthalamic Nucleua, VTA and Substantia Nigra in the Primate Society for Neuroscience Annual Meeting, New Orleans.

Freedman LJ, Insel TR, Smith Y (2000). Subcortical projections of area 25 (subgenual cortex) of the macaque monkey. J Comp Neurol 421: 172-188.

Fudge JL, Haber SN (2001). Bed nucleus of the stria terminalis and extended amygdala inputs to dopamine subpopulations in primates. Neuroscience 104: 807-827.

Georges F, Aston-Jones G (2002). Activation of ventral tegmental area cells by the bed nucleus of the stria terminalis: a novel excitatory amino acid input to midbrain dopamine neurons. J Neurosci 22: 5173-5187.

Goldman-Rakic PS (1999). The physiological approach: functional architecture of working memory and disordered cognition in schizophrenia. Biol Psychiatry 46: 650-661.

Goldman-Rakic PS, Muly III EC, Williams GV (2000). D(1) receptors in prefrontal cells and circuits. Brain Res Brain Res Rev 31: 295-301.

Goldman-Rakic PS, Selemon LD (1997). Functional and anatomical aspects of prefrontal pathology in schizophrenia. Schizophrenia Bull 23: 437-458.

Green MF (1996). What are the functional consequences of neurocognitive deficits in schizophrenia? Am J Psychiatry 153: 321-330.

Groenewegen HJ, Wright CI, Uylings HB (1997). The anatomical relationships of the prefrontal cortex with limbic structures and the basal ganglia. J Psychopharmacol 11: 99-106.

Haber SN (2003). The basal ganglia. In: The Human Nervous System. Paxinos G, Mai JK (eds). Academic Press: New York.

Haber SN, Lynd-Balta E, Mitchell SJ (1993). The organization of the descending ventral pallidal projections in the monkey. J Comp Neurol 329: 111-129.

Hurley KM, Herbert H, Moga MM, Saper CB (1991). Efferent projections of the infralimbic cortex of the rat. J Comp Neurol 308: 249-276.

Karreman M, Moghaddam B (1996). The prefrontal cortex regulates the basal release of dopamine in the limbic striatum: an effect mediated by ventral tegmental area. J Neurochem 66: 589-598.

Keefe KA, Gerfen CR (1999). Local infusion of the (+/-)-a-amino3-hydroxy-5-methylisoxazole-4-propionate/kainate receptor antagonist 6-cyano-7-nitroquinoxaline-2,3-dione does not block D1 dopamine receptor-mediated increases in immediate early gene expression in the dopamine-depleted striatum. Neuroscience 89: 491-504.

Kitano H, Tanibuchi I, Jinnai K (1998). The distribution of neurons in the substantia nigra pars reticulata with input from the motor, premotor and prefrontal areas of the cerebral cortex in monkeys. Brain Res 784: 228-238.

Kolomiets BP, Deniau JM, Glowinski J, Thierry AM (2003). Basal ganglia and processing of cortical information: functional interactions between trans-striatal and trans-subthalamic circuits in the substantia nigra pars reticulata. Neuroscience 117: 931-938.

Kornhuber J (1984). The cortico-nigral projection: reduced glutamate content in the substantia nigra following frontal cortex ablation in the rat. Brain Res 322: 124-126.

Kunzle H (1978). An autoradiographic analysis of the efferent connections from premotor and adjacent prefrontal regions (areas 6 and 9) in macaca fascicularis. Brain Behav Evol 15: $185-234$.

Laruelle M, Abi-Dargham A, van Dyck CH, Gil R, De Souza CD, Erdos J et al (1996). Single photon emission computerized tomography imaging of amphetamine-induced dopamine release in drug free schizophrenic subjects. Proc Natl Acad Sci USA 93: 9235-9240. 
Leichnetz GR, Astruc J (1976). The efferent projections of the medial prefrontal cortex in the squirrel monkey (Saimiri sciureus). Brain Res 109: 455-472.

Manoach D, Press D, Thangaraj V, Searl M, Goff DC, Halpern E et al (1999). Schizophrenic subjects activate dorsolateral prefrontal cortex during a working memory task, as measured by fMRI. Biol Psychiatry 45: 1128-1137.

Manoach DS, Gollub RL, Benson ES, Searl MM, Goff DC, Halpern E et al (2000). Schizophrenic subjects show aberrant fMRI activation of dorsolateral prefrontal cortex and basal ganglia during working memory performance. Biol Psychiatry 48: 99-109.

Maurice N, Deniau JM, Glowinski J, Thierry AM (1999). Relationships between the prefrontal cortex and the basal ganglia in the rat: physiology of the cortico-nigral circuits. J Neurosci 19: 46744681.

McGeorge AJ, Faull RLM (1989). The organization of the projection from the cerebral cortex to the striatum in the rat. Neuroscience 29: 503-537.

Meissner W, Harnack D, Reese R, Paul G, Reum T, Ansorge M et al (2003). High-frequency stimulation of the subthalamic nucleus enhances striatal dopamine release and metabolism in rats. J Neurochem 85: 601-609.

Monakow KH, Akert K, Kunzle H (1978). Projections of the precentral motor cortex and other cortical areas of the frontal lobe to the subthalamic nucleus in the monkey. Exp Brain Res 33: 395-403.

Nambu A, Takada M, Inase M, Tokuno H (1996). Dual somatotopical representations in the primate subthalamic nucleus: evidence for ordered but reversed body-map transformations from the primary motor cortex and the supplimentary motor area. J Neurosci 16: 2671-2683.

Nambu A, Tokuno H, Inase M, Takada M (1997). Corticosubthalamic input zones from forelimb representations of the dorsal and ventral divisions of the premotor cortex in the macaque monkey: comparison with the input zones from the primary motor cortex and the supplementary motor area. Neurosci Lett 239: $13-16$

Öngür D, An X, Price JL (1998). Prefrontal cortical projections to the hypothalamus in macaque monkeys. J Comp Neurol 401: 480-505.

Paquet M, Tremblay M, Soghomonian J-J, Smith Y (1997). AMPA and NMDA glutamate receptor subunits in midbrain dopaminergic neurons in the squirrel monkey: an immunohistochemical and in situ hybridization study. J Neurosci 17: 1377-1396.

Parent A (1986). Comparative Neurobiology of the Basal Ganglia. John Wiley and Sons: New York.

Reep RL, Corwin JV, Hashimoto A, Watson RT (1984). Afferent connections of medial precentral cortex in the rat. Neurosci Lett 44: $247-252$.
Rosales MG, Flores G, Hernandez S, Martinez-Fong D, Aceves J (1994). Activation of subthalamic neurons produces NMDA receptor-mediated dendritic dopamine release in substantia nigra pars reticulata: a microdialysis study in the rat. Brain Res 645: 335-337.

Seeman P, Lee T (1975). Antipsychotic drugs: direct correlation between clinical potency and presynaptic action on dopamine neurons. Science 188: 1217-1219.

Sesack SR, Carr DB (2002). Selective prefrontal cortex inputs to dopamine cells: implications for schizophrenia. Physiol Behav 77: $513-517$.

Sesack SR, Deutch AY, Roth RH, Bunney BS (1989a). Topographical organization of the efferent projections of the medial prefrontal cortex in the rat: an anterograde tract-tracing study with Phaseolus vulgaris leucoagglutinin. J Comp Neurol 290: 213-242.

Sesack SR, Deutch AY, Roth RH, Bunney BS (1989b). Topographical organization of the efferent projections of the medial prefrontal cortex in the rat: an anterograde tract-tracing study with Phaseolus vulgaris leucoagglutinin. J Comp Neurol 290: 213-242.

Sesack SR, Pickel VM (1992a). Prefrontal cortical efferents in the rat synapse on unlabeled neuronal targets of catecholamine terminals in the nucleus accumbens septi and on dopamine neurons in the ventral tegmental area. J Comp Neurol 320: 145-160.

Sesack SR, Pickel VM (1992b). Prefrontal cortical efferents in the rat synapse on unlabled neuronal targets of catecholamine terminals in the nucleus accumbens septi and on dopamine neurons in the ventral tegmental area. J Comp Neurol 320: $145-160$.

Smith Y, Hazrati L-N, Parent A (1990). Efferent projections of the subthalamic nucleus in the squirrel monkey as studied by the PHA-L anterograde tracing method. J Comp Neurol 294: 306-323.

Smith Y, Shink E, Sidibe M (1998). Neuronal circuitry and synaptic connectivity of the basal ganglia. Neurosurg Clin $N$ Am 9: 203-222.

Takagishi M, Chiba T (1991). Efferent projections of the infralimbic (area 25) region of the medial prefrontal cortex in the rat: an anterograde tracer PHA-L study. Brain Res 566: 26-39.

Takahata R, Moghaddam B (2000). Target-specific glutamatergic regulation of dopamine neurons in the ventral tegmental area. J Neurochem 75: 1775-1778.

Van Eden CG, Lamme VA, Uylings HB (1992). Heterotopic cortical afferents to the medial prefrontal cortex in the rat. A combined retrograde and anterograde tracer study. Eur J Neurosci 4: 77-97.

Weinberger DR (1987). Implications of the normal brain development for the pathogenesis of schizophrenia. Arch Gen Psychiatry 44: $660-669$. 